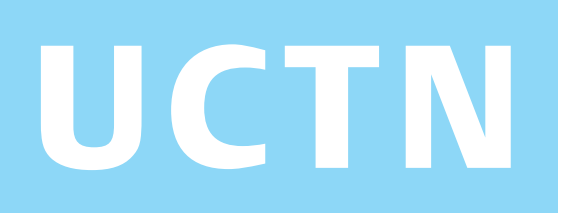

\title{
Colonic spirochetosis associated with dermatomyositis
}
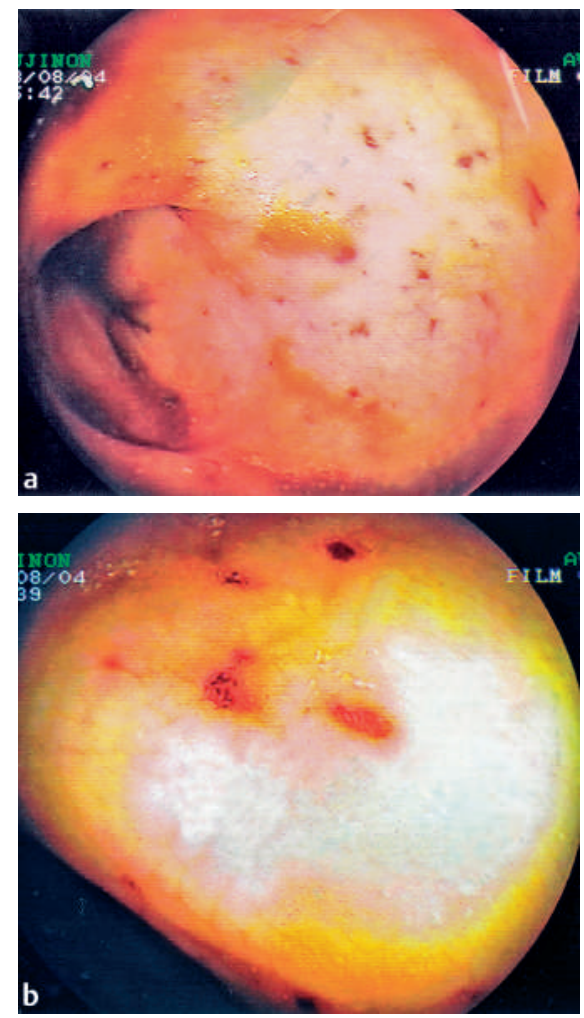

Figure 1 Sigmoidoscopic view of the rectosigmoid region, showing multiple, superficial, punctate mucosal lesions (a), and a close-up view of the same lesions (b).

A 37-year-old man presented with severe myalgia associated with abdominal pain and offensive-smelling diarrhea. He had an erythematous rash on his face, torso, and arms. His creatine kinase was raised at $30145 \mathrm{IU} / 1$ (normal range 33-199 IU/ 1). Electromyography suggested myositis and a muscle biopsy confirmed a diagnosis of dermatomyositis. On abdominal computed tomography he was found to have an edematous large bowel, with an appearance suggestive of inflammatory bowel disease. Fecal microscopy and stool cultures were unrevealing. Flexible sigmoidoscopy showed multiple, punctate hemorrhagic lesions (Figure 1). Biopsies showed a blue fringe along the surface of the colonic epithelium that was suggestive of spirochetosis (with no evidence of cytomegalovirus infection). The patient denied having any occupational contact with animals and he had not been abroad for several years. HIV serology was nega-

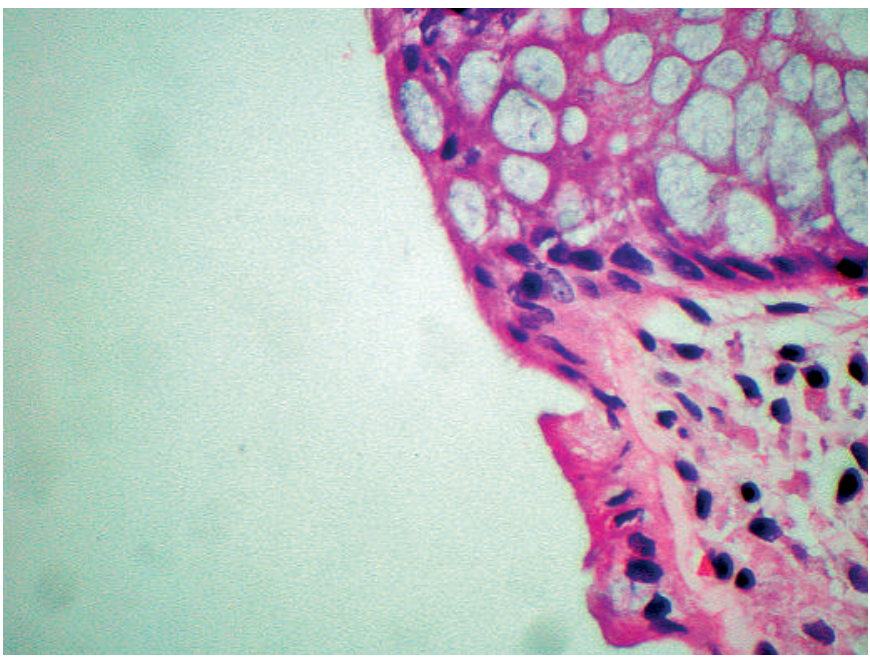

Figure 2 Histological view of a biopsy specimen taken at the repeat sigmoidoscopy, showing colonic mucosa with a fine, violet-blue "fringe" (hematoxylin and eosin stain, original magnification $\times 40$ ).

tive; Borrelia burgdorferi IgG/IgM antibodies and parvovirus B19 IgM antibodies were undetectable.

The patient went on to develop nasal speech and respiratory depression that necessitated the institution of ventilatory support. He was treated with steroids and azathioprine for the dermatomyositis and he made a slow but full recovery after 6 7 weeks. Repeat sigmoidoscopy revealed macroscopically normal mucosa despite the persistence of the histological abnormalities (Figure 2). He remains asymptomatic on follow-up.

Colonic spirochetosis is caused by Gramnegative anaerobic bacteria of the Brachyspira genus (B. aalborgi and B. pilosicoli). B. pilosicoli causes disease in both humans and animals; B. aalborgi affects only humans and higher primates [1]. Intestinal spirochetosis is common in developing countries but the prevalence in the Western world is low (approximately $1 \%$ ), although it remains high in homosexuals and in HIV-positive patients $(30 \%-50 \%)$ [2]. Invasion of spirochetes beyond the surface epithelium is associated with diarrhea, rectal bleeding, and crampy abdominal pains; noninvasive infections are asymptomatic. The diarrhea is usually self-limiting but it is quite common for patients to develop some degree of persistent subclinical infection. Treatment with benzylpenicillin or metronidazole is otherwise effective antimicrobial therapy.

The association of dermatomyositis with spirochetal infection (Borrelia) has already been reported [3]. To the best of authors' knowledge, Brachyspira-associated dermatomyositis has not been previously described in the English-language literature.

\section{Endoscopy_UCTN_Code_CCL_1AD_2AZ}

\section{A. Koulaouzidis' ${ }^{1}$, S. Campbell ${ }^{2}$,}

S. Ahmed ${ }^{1}$, S. Prados ${ }^{1}$, W. C. Tan ${ }^{1}$

${ }^{1}$ Department of Gastroenterology, Warrington Hospital, Warrington, Cheshire, United Kingdom

2 Department of Gastroenterology, Royal University Liverpool Hospital, Liverpool, United Kingdom. 
${ }^{1}$ Smith JL. Colonic spirochetosis in animals and humans. J Food Prot 2005; 68: $1525-$ 1534

${ }^{2}$ Alsaigh N, Fogt F. Intestinal spirochetosis: clinicopathological features with review of the literature. Colorectal Dis 2002; 4: $97-$ 100

${ }^{3}$ Horowitz HW, Sanghera K, Goldberg N et al. Dermatomyositis associated with Lyme disease: case report and review of Lyme myositis. Clin Infect Dis 1994; 18: 166-171

\section{A. Koulaouzidis, MRCP}

Warrington Hospital

Lovely Lane

Warrington WA5 1QG

United Kingdom

Fax: $\quad$ +44-1925-662042

Email: akoulaouzidis@hotmail.com 